\title{
Thermoelectric effect in very thin film Pt/Au thermocouples
}

\author{
M. C. Salvadoria), A. R. Vaz, F. S. Teixeira, M. Cattani \\ Institute of Physics, University of São Paulo, C.P. 66318, CEP 05315-970, \\ São Paulo, SP Brazil
}

and

I. G. Brown

Lawrence Berkeley National Laboratory, 1 Cyclotron Road

Berkeley, California 94720, USA

\begin{abstract}
The thickness dependence of the thermoelectric power of Pt films of variable thickness on a reference Au film has been determined for the case when the Pt film thickness, $t$, is not large compared to the charge carrier mean free path, $\ell$, that is, $t \square \ell$. Pt film thicknesses down to $2.2 \mathrm{~nm}$ were investigated. We find that $\Delta S_{F}=S_{B}-S_{F}$ (where $S_{B}$ and $S_{F}$ are the thermopowers of the Pt bulk and film, respectively) does not vary linearly as $1 / t$ as is the case for thin film thermocouples when the film thickness is large compared to the charge carrier mean free path.
\end{abstract}

Keywords: Thermopower effect; Thin-film platinum-gold thermocouples.

\footnotetext{
${ }^{a)}$ Electronic mail: mcsalvadori@if.usp.br
} 
Thin film thermocouples (TFTC) provide a sensitive way of making accurate and fast (down to $\sim 1 \mu$ s response time) surface temperature measurements. They have the advantages of intimate thermal contact with the surface, low thermal inertia (mass $\sim 10^{-4} \mathrm{~g}$ ), high spatial resolution, and low cost. Development of TFTCs has been vigorous in recent years [1], prompted in large part by the need of the microelectronics industry for TFTCs with eversmaller dimensions. However, when the film thickness becomes comparable to the mean free path of the charge carriers, all transport processes are expected to exhibit size effects [2-9]. Extensive electric and galvanomagnetic studies of thin films of alkali and noble metals have established the existence of size effects, important from both fundamental and technical points of view.

A body of experimental work [9-12] on the electrical conductivity $\sigma_{F}$ of thin films with thickness $t>>\ell$, the charge-carrier mean free path, has shown that the ratio $\sigma_{F} / \sigma_{B}$ is well described by $\sigma_{F} / \sigma_{B}=1-c / t$, where $\sigma_{B}$ is the bulk conductivity and $c$ is a constant. With the same precision the resistivity ratio $\rho_{F} / \rho_{B}$ is given by $\rho_{F} / \rho_{B}=1+c / t$. The $1 / t$ variation is in good agreement with the semiclassical predictions of Fuchs [2]:

$$
\sigma_{F} / \sigma_{B} \approx 1-(3 / 8)(1-p) \ell / t,
$$

where the scattering coefficient, $p$, is the fraction of energy lost by the charge carriers when reflected by the film surface. Since $\rho=1 / \sigma$, Eq. (1) can be written in terms of the resistivity ratio $\rho_{F} / \rho_{B} \approx 1+(3 / 8)(1-p) \ell / t$.

According to Justi et al. [3] and Mayer [4], when Eq. (1) is obeyed the thermoelectric power $S_{F}$ of pure metal thin films with thickness $t>>\ell$ is given by

$$
S_{F}=S_{B}[(1-(3 / 8) \ell / t)(1-p) U /(1+U)], \quad(t>>\ell)
$$

where $U=(\partial \ln \ell(E) / \partial \ln E)_{E=\xi}, E$ is the electron energy, $\xi$ is the Fermi energy, and the bulk thermoelectric power $S_{B}$ is given by

$$
S_{B}=-\left(\pi^{2} / 3 e\right)\left(k^{2} T / \xi\right)(U+1)
$$


wherer $k$ is Boltzmann's constant, $T$ the absolute temperature, and $e$ the electron charge.

From Eqs. (2) and (3) we obtain

$$
\begin{array}{rlr}
\Delta S_{F}=S_{B}-S_{F} & =S_{B}(3 / 8)[(\ell / t)(1-p) U /(1+U)] & (t>>\ell) \\
& =-\left(\pi^{2} / 8 e\right)\left(k^{2} T / \xi\right)[\ell U(1-p) / t] \\
& =-9.2 \times 10^{-3}(T / \xi)[\ell U(1-p) / t] \quad(\mu \mathrm{V} / \mathrm{deg})
\end{array}
$$

If $\Phi_{F}$ and $\Phi_{B}$ are the thermopowers of a thin-film and a thick-film of Pt with respect to a thick standard film, respectively, then

$$
\Delta S_{F}=\Phi_{B}-\Phi_{F} .
$$

From Eq. (4), a plot of $\Delta S_{F}$ vs $1 / t$ yields a straight line through the origin, the slope of which determines $\ell U(1-p)$. For polycrystalline films [8] $p \approx 0$ and $\ell$ can be calculated using the conductivity relation $\sigma=\left(n e^{2} / m v_{F}\right) \ell$, where $n$ is the number of conducting electrons per unit volume, $v_{F}$ is the Fermi velocity, and $m$ is the electron mass. Thus we can determine the value of $U$.

Thermocouple studies of films of alkali metals [13], bismuth [8] and cobalt [5] have shown agreement with Eq. (2) and a value of $U=2$, i.e., $\ell \sim E^{2}$, in accord with the Bloch quantum theory of electrical conduction in metals. Huebener [14] obtained $U=-0.53 \pm 0.19$ for thin gold foils at temperatures between 77 and $296 \mathrm{~K}$. Worobey et al. [6] have obtained $U$ $=-0.61 \pm 0.20$ for thin gold films above $200 \mathrm{~K}$. Chopra et al. [8] obtained $U=-18.7$ for copper thin films at $T=483 \mathrm{~K}$. Thornburg and Wayman [15] found $U \approx-2.2$ for Au-Ni thinfilms. Scarioni and Castro [1] found $U=-49.4$ for $\mathrm{Fe}-\mathrm{Cu} \mathrm{Ni}$ alloy thin films in the temperature range 296 - 473 K. Studies performed by Prasad et al. [16] and by Schepis et al. [17] of the thermoelectric power of thin iron films showed the predicted $1 / t$ thickness dependence. 
For all of the above experimental work [1,5,6,8,14-17] the film thickness $t$ was much greater than the electron mean free path $\ell, t>>\ell$, and plots of the measured thermopower $\Delta S_{F}$ as a function of $1 / t$ yield a straight lines through the origin in agreement with Eq. (4). The $U$ values for metals, however, are very different from $U=2$ predicted by the free electron hypothesis of Bloch.

We have studied the thermopower of TFTCs when $t \square \ell \ell$. We have measured $\Delta S_{F}$ for $\mathrm{Pt} / \mathrm{Au}$ thermocouples with very thin Pt film and thick $\mathrm{Au}$ film as reference. For the $\Delta S_{F}$ measurements the Pt film thickness $t$ was varied over the range $2.2 \leq t \leq 24.5 \mathrm{~nm}$. The mean free path for $\mathrm{Pt}$ is $\ell_{\mathrm{Pt}} \approx 10 \mathrm{~nm}$, and thus the $t / \ell$ range spanned is $0.22 \leq t / \ell \leq 2.45$. We find that $\Delta S_{F}$ does not vary linearly as $1 / t$ as for the $t>>\ell$ case. Interestingly, some indication of this nonlinearity can be seen, for instance, in the paper of Chopra et al. [8] for copper films with thickness smaller than $25 \mathrm{~nm}$.

The Pt and Au films were formed using a filtered vacuum arc plasma deposition method that has been fully described elsewhere [18]. Briefly, a repetitively-pulsed vacuum arc plasma gun equipped with either a $\mathrm{Au}$ or Pt cathode is used to form a dense metal (Au or Pt) plasma, which is then transported through a $90^{\circ}$ bent solenoidal magnetic field to remove any solid particulates (cathode debris) from the plasma stream. The plasma is then allowed to deposit on a substrate that is positioned near the exit of the solenoid filter. Thin film samples of different thicknesses can readily be prepared by varying the number of plasma pulses used for the deposition. For the work described here the plasma pulses were $5 \mathrm{~ms}$ long and the repetition rate was $1 \mathrm{pulse} / \mathrm{sec}$. The substrates used for film deposition were ordinary glass microscope slides of dimension $25 \times 75 \mathrm{~mm}^{2}$. The geometry used for all samples is shown in Fig. 1. The Au film strips were the same thickness for all samples, and the thickness of the Pt film was varied for the different samples. We prepared a set of samples in which the Au films were of thickness $141 \pm 2 \mathrm{~nm}$ and the Pt films were of thicknesses from 2.2 to $166 \mathrm{~nm}$. 
Importantly, we note that the vacuum arc plasma deposition method that we have used is an energetic deposition, with the depositing Pt ion flux having a directed ion energy of $\sim 100 \mathrm{eV}$ [19], resulting in films that are void free and fully dense [20].

Measurements were made with the hot junction immersed in oil inside a calorimeter at temperature $T_{1}$ varied over the range $297 \leq \mathrm{T}_{1} \leq 383 \mathrm{~K}$, and with the cold junction immersed in a large thermal reservoir filled with oil maintained at a constant temperature $T_{2}=297 \mathrm{~K}$ (ambient), taken as the reference temperature. The hot-junction temperature was monitored with a digital thermometer using a type- $K$ (cromel-alumel) thermocouple. The thermoelectric voltage $V$ was measured at the cold junction.

The measured thermoelectric powers $S$ are shown in Table I, and these data are plotted in Fig. 2. The continuous line shown is a best-fit to the experimental results, given by $S=3.69[1-\exp (-0.046 t)]$. It can be seen that $S$ reaches a constant for $t>100 \mathrm{~nm}$ at a maximum value $S_{\max }=3.62 \mu \mathrm{V} / \mathrm{K}$. (The thermoelectric power for bulk Au/Pt thermocouple is $6.5 \mu \mathrm{V} / \mathrm{K}[21]$; this bulk value is reached only for films much thicker than those used here).

Table I also shows $\Delta S_{F}(t)=S_{\max }-S(t)$, and these data are plotted in Fig. 3 as a function of $1 / t$. The experimental results are represented by solid squares, and the continuous line is a best-fit to the experimental results, given by the function $\Delta S_{F}=0.194+26.7(1 / t)-93.6(1 / t)^{2}$ $+112.0(1 / t)^{3}$. The straight, dashed line shows the linear behavior of $\Delta S_{F}$ as a function of $1 / t$ as expected for films thick compared to about $20 \mathrm{~nm}, t>>\ell$, as per the discussion above and Eq. (4). The figure clearly shows that the thermopower $\Delta S_{F}$ does not vary linearly with $1 / t$ for very thin Pt films, of thickness less than about $20 \mathrm{~nm}$. This is in accord with the theoretical predictions described above, a linear relationship is expected for $t>>\ell$, but not for very thin films as explored here, $t \square \ell$. For these very thin films, the electrical resistivity ratio $\rho_{F} / \rho_{B}$ is not correctly given by $\rho_{F} / \rho_{B} \approx 1+(3 / 8)(1-p) \ell / t$ as in Eq. (1). In previous work [22] we have 
shown that the ratio $\rho_{F} / \rho_{B}$ for Pt films with thickness in the range $1.31 \leq t \leq 11.66 \mathrm{~nm}$ is given by $\rho_{F} / \rho_{B}=1+6.5 / t+140 / t^{9}$. Studies of the electrical resistivity of metallic films [23, 24] have revealed that this behavior is due to quantum size effects that become relevant when $\ell \geq t$. Eqs. (1)-(4) were derived using a semiclassical approach [2-4] that is applicable only when quantum size effects are negligible, i.e., when $t>>\ell$, resulting in $\Delta S_{F}$ varying linearly with $1 / t$. To explain the observed nonlinear relationship it would be necessary to adopt a quantum mechanical formalism to calculate the electrical resistivity and the thermoelectric power.

\section{Acknowledgements}

The authors thank FAPESP and the CNPq for financial support. 


\section{References}

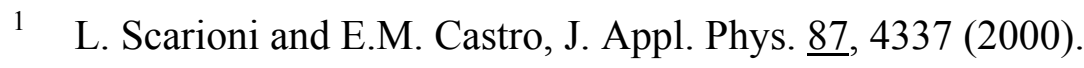

2 K. Fuchs, Proc. Cambridge Phil. Soc. 34, 100 (1938).

3 E. Justi, K. Kohler and G. Lautz, Z. Naturforsch. $\underline{6 A}, 456$ and 544 (1951).

4 H. Mayer, Physik dünne Schichten, (Wissenschaftlische-Verlagsgesellschaft MBH, Stuttgart, 1955), vol. 2, p. 232.

5 F. Savornin, Electric and Magnetic Properties of Thin Films (Leuven Symposium, 1961).

6 W. Worobey, P. Lindenfeld and B. Serin, Basic Problems in Thin-Film Physics, R. Niedermayer and H. Mayer, Eds. (Vandenhoeck-Ruprecht, Gottingen, 1965), p. 601.

7 R. Marshall, L. Atlas and T. Putner, J. Sci. Instr. 43, 144 (1966).

8 K.L. Chopra, S.K. Bahl and M.R. Randlett, J. Appl. Phys. 39, 1525 (1968) and references therein.

9 C.R. Tellier and A.J. Tosser, Size Effects in Thin Films, (Elsevier, Amsterdam,1982) and many references therein.

10 M. Jalochowski, E. Bauer, H. Knoppe and G. Lilienkamp, Phys. Rev. B 또, 13607 (1992).

11 E.V. Barnat, D. Nagakura, P.I. Wang and T.M. Lu, J. Appl. Phys. 91, 1667 (2002).

12 S.M. Rossnagel and T.S. Kuan, J. Vac. Sci. Technol. A 20, 1911 (2002).

13 R. Nossek and Z. Naturforsch. 16A, 1162 (1961).

14 R.P. Huebner, Phys. Rev. 136A, 1740 (1964).

15 D.D. Thornburg and C.M. Wayman, J. Appl. Phys. $\underline{40}, 3007$ (1969).

16 R.D.G. Prasad, G.M. Rao and S. Mohan, J. Mat. Sci. Lett. $\underline{9}, 650$ (1990).

17 R.S. Schepis, L.J. Matienzo, F. Emmy, W. Unertl and K. Schröeder, Thin Solid Films 251, 99 (1994).

18 M.C. Salvadori, I.G. Brown, A.R. Vaz, L.L. Melo and M. Cattani. Phys. Rev. B $\underline{67}$, 
153404 (2003).

19 A. Anders and G. Yushkov, J. Appl. Phys. $\underline{91}, 4824$ (2002).

20

I.G. Brown, "Cathodic Arc Deposition of Films", in Annual Review of Materials Science, Vol. 28 (Annual Reviews, Inc., Palo Alto, CA, 1998).

21 J.W. Dally, W.F. Riley and K.G. McConnell, Instrumentation for Engineering Measurements, (Wiley, New York,1984).

22 M.C. Salvadori, A.R. Vaz, J.C. Farias and M. Cattani. Surf. Rev. Lett. 11, 223 (2004).

23 G. Fishman and D. Calecki. Phys. Rev. Lett. 62, 1302 (1989) and references therein.

24 G. Palasantzas, Y.P. Zhao, G.C. Wang, T.M. Lu, J. Barnas and J.T.M. de Hosson. Phys. Rev. B61, 11109 (2000) and references therein. 


\section{TABLE I.}

Measured thermoelectric power $S$ for samples of different Pt film thickness $t$, for fixed Au film thickness of $141 \mathrm{~nm}$.

\begin{tabular}{ccc}
\hline $\begin{array}{c}\text { Thickness, } t \\
(\mathrm{~nm})\end{array}$ & $\begin{array}{c}S(t) \\
(\mu \mathrm{V} / \mathrm{K})\end{array}$ & $\begin{array}{c}\Delta S_{F}(t)=3.62-S(t) \\
(\mu \mathrm{V} / \mathrm{K})\end{array}$ \\
\hline 2.2 & 0.181 & 3.46 \\
4.1 & 0.643 & 2.00 \\
5.0 & 1.23 & 2.41 \\
5.9 & 1.03 & 2.61 \\
9.9 & 1.82 & 1.82 \\
20.8 & 2.03 & 1.61 \\
24.5 & 2.40 & 1.24 \\
90.1 & 3.79 & - \\
166 & 3.62 & - \\
\hline
\end{tabular}




\section{Figures Captions}

Fig. 1: The thermocouples are formed of and Pt and Au strips, $6 \mathrm{~mm}$ wide, on glass microscope slides. The thermoelectric voltage $V$ is measured as indicated.

Fig. 2: Measured thermopower $S$ as a function of Pt film thickness $t$. The thickness of the Au film was fixed at $141 \mathrm{~nm}$. The line is a best fit to the data.

Fig. 3: Measured $\Delta S_{F}(t)=\Phi_{B}-\Phi_{F}$ as a function of the inverse Pt film thickness $1 / t$. The full line is a best fit to the data, and the dashed line shows the linear behavior of $\Delta S_{F}$ which as expected for films much thicker than $20 \mathrm{~nm}$. 


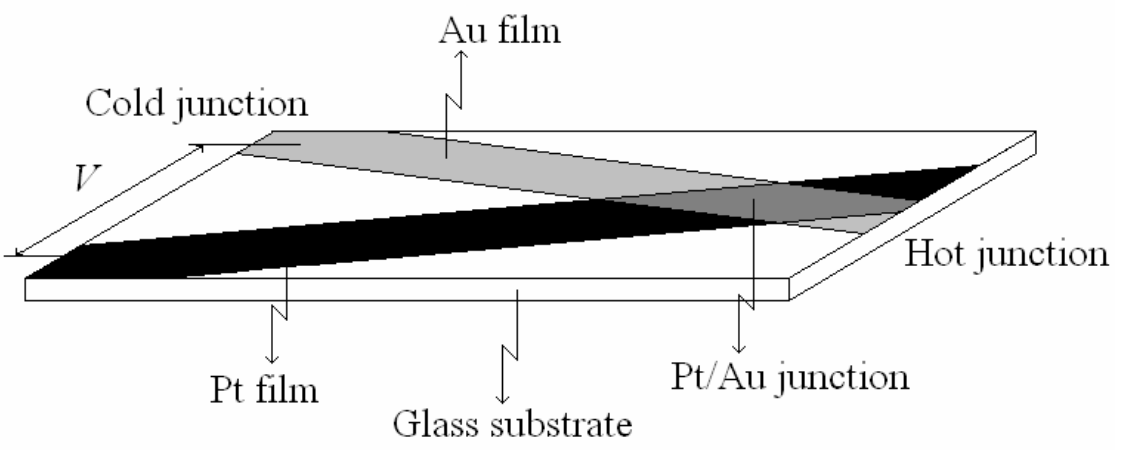

Fig. 1 


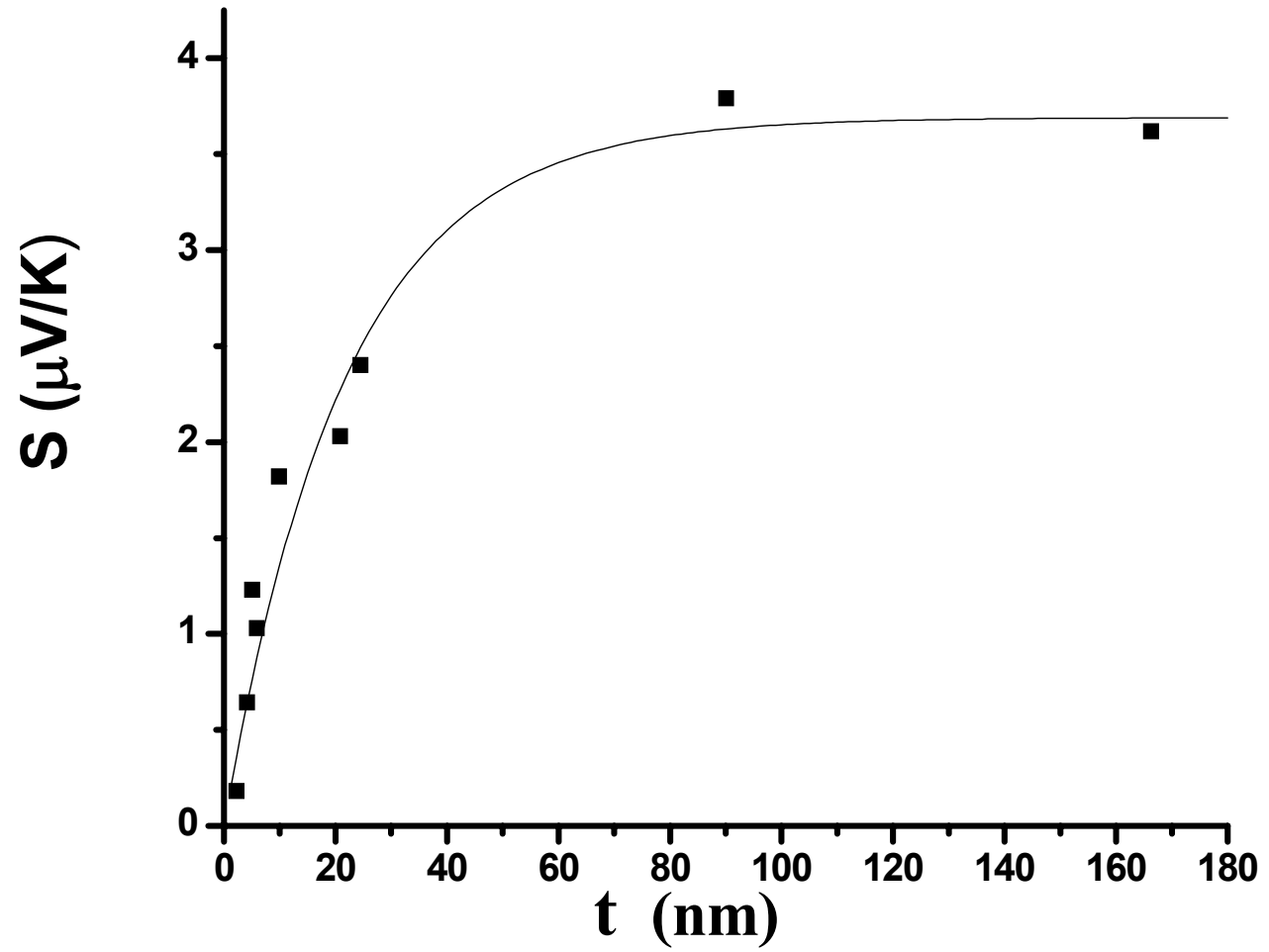

Fig. 2 


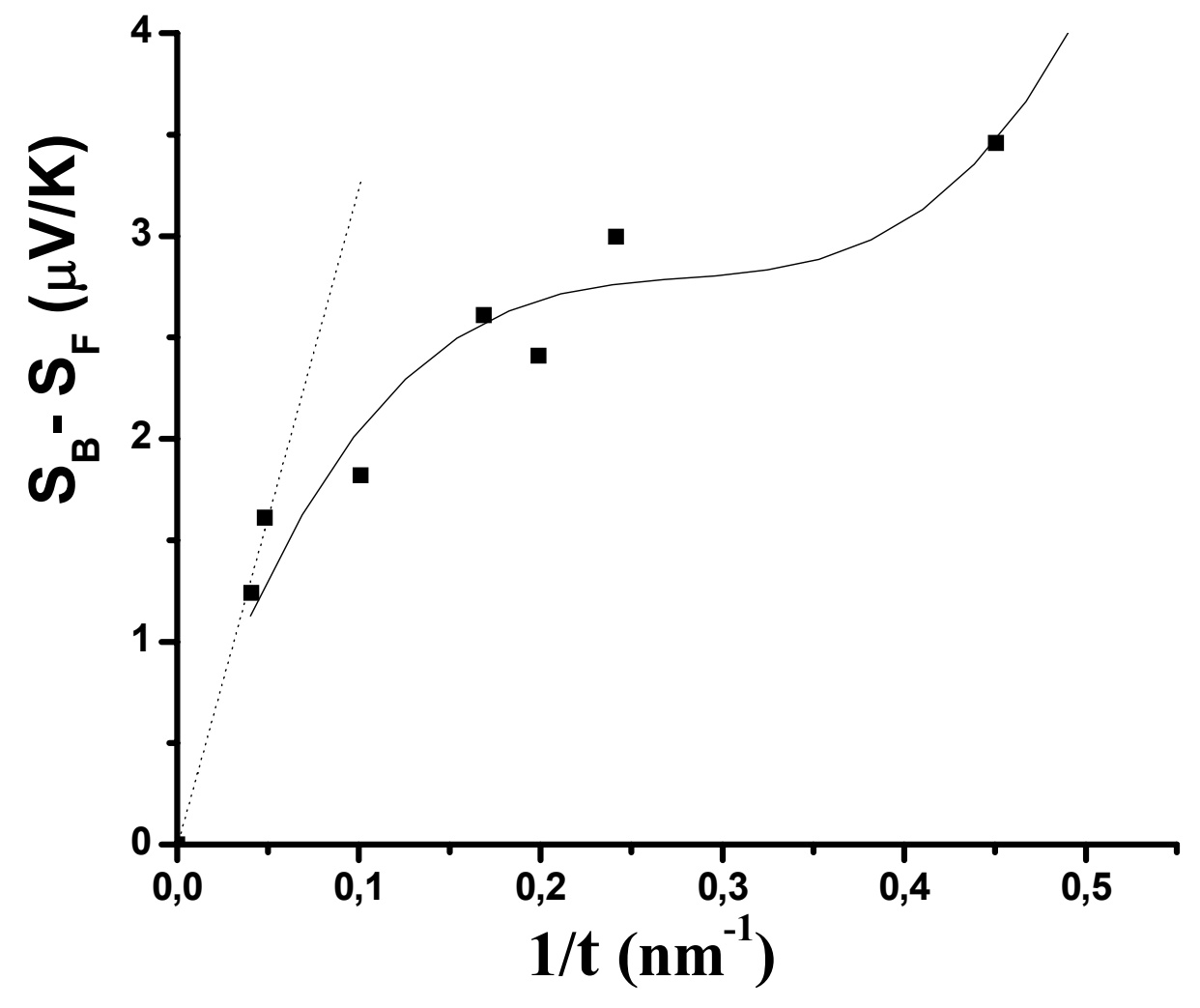

Fig. 3 